\title{
Pago por desempeño en el sistema colombiano de salud
}

\section{Pay for performance in Colombian healthcare}

Iouri Gorbanev ${ }^{1}$, Ariel Cortes ${ }^{2}$, Sergio Torres ${ }^{1}$ y Francisco Yepes ${ }^{2}$

1 Departamento de Administracion, Facultad de Ciencias Económicas y Administrativas, Pontificia
Universidad Javeriana. yurigor@javeriana.edu.co . storres@javeriana.edu.co
2 Postgrados en Administracion de salud, Facultad de Ciencias Económicas y Administrativas,
Pontificia Universidad Javeriana, ariel.cortes@javeriana.edu.co, fyepes@javeriana.edu.co

Recibido 14 Junio 2011/Enviado para Modificación 3 Octubre 2011/Aceptado 25 Octubre 2011

\section{RESUMEN}

Objetivo Describir el alcance y las formas de uso de Pago por Desempeño en la cadena de salud Colombiana

Métodos Un estudio observacional descriptivo basado en las entrevistas y encuestas aplicadas a los directivos de las Aseguradoras en salud en Bogotá, Colombia. Para interpretar los resultados, los autores se apoyan sobre la Teoría de Costos de Transacción.

Resultados Pago por Desempeño se usa por las Aseguradoras del régimen Contributivo en el nivel ambulatorio, básicamente en Promoción y Prevención. Pago por Desempeño no se usa en el nivel hospitalario. Las Aseguradoras Subsidiadas no utilizan Pago por Desempeño. De igual manera, el pago por Desempeño no se usa en el caso de los llamados vinculados.

Conclusiones El alcance de uso de Pago por Desempeño en Colombia es limitado. La practica colombiana solo parcialmente valida el planteamiento de la Teoría de Costos de Transacción sobre los atributos de formas de gobernación, uno de los cuales es la intensidad de incentivos.

Palabras Clave: Organización, administración, motivación, sistemas de salud (fuente: DeCS, BIREME).

\section{ABSTRACT}

Objective Describing the extent and forms of use of pay for performance (P4P) in Colombian healthcare.

Methods This was a descriptive study based on interviews and surveys of health insurance agency managers in Bogotá, Colombia. The authors relied on transaction cost theory to interpret the results.

Results P4P was found to be used by contribution scheme insurers in an outpatient setting, basically in promotion and prevention; $\mathrm{P} 4 \mathrm{P}$ is not being used in a hospital setting. Subsidized scheme insurers do not use P4P. Similarly, P4P is not being used in the case of so called associated users. 
Conclusions P4P use in Colombia is limited. Colombian practice only partially validates the transaction costs theory approach to governance model attributes, one of which is incentive intensity.

Key Words: Organization, administration, reimbursement, incentive, healthcare (source: MeSH. NLM).

$\mathrm{E}$ 1 trabajo reporta los resultados del estudio sobre el uso del Pago por Desempeño (Pay for Performance, o P4P) en la cadena de salud colombiana. El Pago por desempeño despierta cierto entusiasmo porque teóricamente permite alinear el esfuerzo del Proveedor con los objetivos de la Aseguradora y lograr el alto desempeño de la cadena de salud. A pesar de su intuitivo atractivo, no es fácil implantar el Pago por desempeño para alinear los intereses de la Aseguradora y Prestadora. En un primer momento, no existe la claridad conceptual. Surgen dudas y discusiones acerca de cómo se entiende el desempeño. Es un concepto amplio que incluye tanto la calidad como la eficiencia. El uso común del término Pago por desempeño abarca estos dos ámbitos con el énfasis en la calidad.

En estas condiciones la técnica administrativa mencionada necesita una mayor definición. Por ahora es una etiqueta común que agrupa diferentes esquemas de incentivos monetarios. Se diferencian entre sí en cuanto a los indicadores de desempeño, criterios de calidad, tipo y magnitud de pago, formulas que determinan el pago, la naturaleza de transacciones en que se usan. No hay acuerdo en cuanto al efecto que ejerce el Pago por desempeño. La literatura evidencia resultados mixtos que van de los positivos a los negativos pasando por nulos. Es un resultado previsible porque el estudio de los incentivos está en su fase inicial y falta mucho para entender sus determinantes de éxito y el diseño óptimo de incentivos $(1,2)$.

A pesar de la falta de una evidencia contundente a favor de Pago por desempeño y la ausencia de los instrumentos administrativos aplicables en la práctica, los administradores de salud cada vez más dirigen sus miradas hacia esta técnica (3).

La literatura colombiana no reporta trabajos sobre el Pago por desempeño en salud. Es sorprendente porque en Colombia funciona un mercado regulado de servicios de salud que funciona con base en los incentivos. Por eso se 
hace interesante preguntar, cual es el alcance de uso de este mecanismo en Colombia. Para contestar esta pregunta, se hace un estudio exploratorio del uso de Pago por desempeño por las Aseguradoras en sus relaciones con las Prestadoras de salud en Colombia.

En lo que sigue, el trabajo está dividido en 4 secciones. La primera sección presenta el resumen de literatura, la segunda, los instrumentos y métodos, la tercera, resultados, la cuarta, discusión y conclusiones.

Conceptualmente, P4P es un incentivo monetario. El incentivo se define como un factor que motiva la persona a preferir cierto curso de acción a otros. El concepto de incentivo se ha trabajado desde tiempos remotos por el filósofo chino Mozi en el siglo V a. C (4), A. Smith (5), C. Marx (4), F. Taylor (6).

Recientemente, la teoría de la agencia se constituyó en la teoría económica de incentivos. Recomienda alinear los intereses del agente y principal por medio de incentivos que establecen el balance entre el riesgo y el incentivo. $\mathrm{Si}$ el agente recibe solo el pago fijo, no corre riesgo pero tampoco tiene incentivos. Si el agente recibe solo el pago variable según su desempeño, tiene el incentivo pero queda expuesto al riesgo. El contrato eficiente esta en un punto entre estos dos extremos (7). Un obstáculo en este camino es la complejidad de trabajo. Cuando el resultado de trabajo es tangible y cuantificable, es fácil relacionar el incentivo con el resultado. Cuando el trabajo es complejo o el agente es responsable de varias tareas, el principal tiene que contentarse con monitorear la acción del agente (8) y preocuparse sobre cómo el incentivo, diseñado para realizar una tarea, afecta otras tareas (9). "El énfasis en la eficiencia puede dañar la calidad clínica. El énfasis en la calidad y eficiencia harán que la atención no va a estar centrada en el usuario" (10).

La literatura identifica numerosas situaciones cuando el P4P tiene efectos paradójicos. Fehr y Rockenbach reportan el efecto dañino del incentivo negativo (castigo) sobre la cooperación altruista (11). El incentivo crea la dependencia y puede causar el daño cuando es retirado (12). El efecto del incentivo depende de una serie de factores ajenos como el grado de la integración vertical entre el proveedor y comprador del servicio, de las economías de escala, de si los proveedores (en este caso, médicos) funcionan como independientes o en grupos, de las relaciones de propiedad, del monitoreo, de la capacidad administrativa (13). El mismo hecho de que una actividad 
está sujeta a la medición, influencia la conducta de la persona que la ejecuta (14). La asignación de la propiedad y la decisión sobre la integración vertical no es indiferente para la motivación (15). El incentivo legítimo de reducir los costos puede llevar a la prestadora de salud a elevar las barreras de acceso (16).

La teoría que permite entender P4P en toda su complejidad es TCT cuyos conceptos se resumen en la Tabla 1.

Tabla 1. Conceptos de TCT

\begin{tabular}{|c|c|c|}
\hline Concepto & Definición & Relación con el incentivo \\
\hline Transacción & Intercambio de bienes o servicios & \\
\hline Incertidumbre & $\begin{array}{l}\text { Situación cuando se conocen las } \\
\text { alternativas pero no se conoce su } \\
\text { distribución de probabilidad }\end{array}$ & $\begin{array}{l}\text { Explica costos de } \\
\text { transacción }\end{array}$ \\
\hline Frecuencia & $\begin{array}{l}\text { Frecuencia con que se realiza la } \\
\text { transacción }\end{array}$ & $\begin{array}{l}\text { Explica costos de } \\
\text { transacción }\end{array}$ \\
\hline Especificidad de activos & $\begin{array}{l}\text { Grado en que un activo puede ser } \\
\text { re direccionado a otro uso }\end{array}$ & $\begin{array}{l}\text { Explica costos de } \\
\text { transacción }\end{array}$ \\
\hline $\begin{array}{l}\text { Costos de transacción en } \\
\text { el mercado }\end{array}$ & $\begin{array}{l}\text { Costos de uso del mecanismo de } \\
\text { precios }\end{array}$ & $\begin{array}{l}\text { Obligan a los actores a } \\
\text { buscar forma de } \\
\text { gobernación adecuada }\end{array}$ \\
\hline Forma de gobernación & $\begin{array}{l}\text { Ambiente institucional en que } \\
\text { transcurre la transacción. Existen } \\
\text { tres formas de gobernación: } \\
\text { Mercado, Hibrido Jerarquía }\end{array}$ & $\begin{array}{l}\text { Cada forma de gobernación } \\
\text { posee los atributos: } \\
\text { intensidad de incentivos, } \\
\text { intensidad de controles } \\
\text { administrativos, el contrato. }\end{array}$ \\
\hline Mercado & Compra - venta episódica & $\begin{array}{l}\text { Incentivos de alta potencia; } \\
\text { Control administrativo nulo; } \\
\text { Contrato clásico de compra }\end{array}$ \\
\hline Hibrido (alianza) & Relación especial de largo plazo & $\begin{array}{l}\text { Incentivos moderados; } \\
\text { Control administrativo } \\
\text { moderado, Contrato } \\
\text { neoclásico relacional }\end{array}$ \\
\hline Jerarquía (organización) & $\begin{array}{l}\text { Relación de autoridad y } \\
\text { obediencia }\end{array}$ & $\begin{array}{l}\text { Incentivos de baja potencia; } \\
\text { Control administrativo alto; } \\
\text { Relación contractual de } \\
\text { empleo }\end{array}$ \\
\hline
\end{tabular}

Fuente: Elaboración propia con base en Williamson (17)

Los citados arriba estudios de Frolich y Petersen hacen resúmenes sistemáticos de la literatura sobre P4P en salud publicada entre 1980-2005; Van Herck, de la literatura publicada entre 1990 y 2009.

La literatura no reporta estudios sobre el uso de P4P en la cadena de salud en Colombia.

\section{MÉTODOS}

Es un estudio exploratorio que pretende describir la situación con el P4P. 
Durante 2009 se elaboró un cuestionario que contenía preguntas diseñadas con base en las variables encontradas en la literatura sobre el P4P, un glosario de conceptos utilizados y el compromiso ético de los investigadores.

En un primer momento, se preguntaba si la Aseguradora utilizaba P4P en algún nivel de atención (ambulatorio u hospitalario). Si la respuesta era positiva, se preguntaba sobre los servicios contratados bajo el esquema de P4P, y formas de pago (por evento, por paquete, per cápita); sobre los motivos de uso de P4P (calidad, eficiencia, imagen y posicionamiento); sobre la forma de pago del incentivo (bonificación variable, bonificación fija, sanción, aumento del volumen de compra); sobre qué cantidad de servicios (en dinero) estaba contratada bajo el esquema de P4P; qué porcentaje del pago habitual constituía el incentivo; el tipo de indicadores que se usaban para constatar el cumplimiento de objetivos (resultado, proceso, estructura); el mecanismo que se usaba para constatar el logro de objetivos (desempeño individual de cada prestadora y torneo, o comparación entre varias Prestadoras).

La prueba de fiabilidad del instrumento realizada con SPSS, arrojó un alfa de 0,899 .

Durante 2009-2010 fueron contactadas por correo y telefónicamente 17 EPS del régimen Contributivo, a las cuales respondieron 5, y 8 EPS del régimen Subsidiado, a las cuales 3 aceptaron participar en el estudio. También se hicieron entrevistas en las Secretarias de Salud de Bogotá y Cundinamarca que manejan el régimen de las personas vinculadas. Los investigadores fueron recibidos por los gerentes, directores médicos o jefes de contratación quienes contestaron la encuesta durante la entrevista. Los investigadores aprovecharon la entrevista para profundizar en el tema. En un caso la encuesta fue contestada sin presencia de investigadores. Todos los formularios se encontraron validos. Los formularios fueron tabulados y la base de datos que fue procesada con métodos de estadística descriptiva.

\section{RESULTADOS}

De 8 EPS, 5 usan P4P. Todas las 5 pertenecen al Régimen Contributivo. Todas las 3 que no usan P4P, pertenecen al Régimen Subsidiado. Las Secretarias de Salud de Bogotá y Cundinamarca que manejan el régimen de las personas vinculadas, no usan P4P. La Tabla 2 resume los niveles de atención donde se usa P4P. 
Se identificaron tres motivos básicos de uso de P4P y las combinaciones de los mismos que se indican en la Figura 1.

El P4P se aplica solo en los servicios ambulatorios, es decir, para contratar los servicios relativamente baratos que no pesan más de $20 \%$ en la facturación total de las Aseguradoras. Más aun: la mayoría de las Aseguradoras utiliza $\mathrm{P} 4 \mathrm{P}$ solo en Promoción y Prevención (PyP).

Tabla 2. Niveles de atención donde se usa P4P, forma de pago habitual y forma de pago del incentivo

\begin{tabular}{ccc}
\hline $\begin{array}{c}\text { Forma de pago } \\
\text { habitual }\end{array}$ & IPS que usan P4P a nivel Ambulatorio & $\begin{array}{c}\text { IPS que usan P4P a } \\
\text { nivel Hospitalario }\end{array}$ \\
\hline Capitación & $\begin{array}{c}\text { PyP } \\
\text { (Pago variable } \\
\text { desempeño) }\end{array}$ & $\begin{array}{c}\text { Otros servicios } \\
\text { (Pago variable } \\
\text { según el } \\
\text { desempeño) }\end{array}$ \\
Pago por servicio & $\begin{array}{c}1 \text { (Pago variable } \\
\text { según el } \\
\text { desempeño) }\end{array}$ & $\begin{array}{c}1 \text { (Modificación del } \\
\text { volumen de compra } \\
\text { según el }\end{array}$ \\
\hline
\end{tabular}

Fuente: Elaboración propia con base en los hallazgos

Figura 1. Combinaciones de motivos para usar P4P. Los números reflejan la cantidad de Aseguradoras que manifestaron tener cierta combinación de motivos

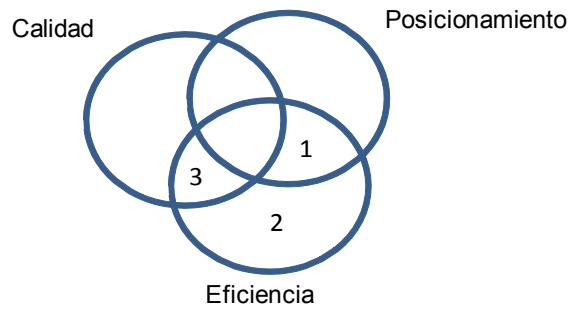

El tamaño de incentivo como proporción del pago habitual por cierto servicio típicamente es de $5 \%$ y nunca supera $15 \%$.

Para evaluar el desempeño de las Prestadoras, dos Aseguradoras utilizan la combinación de Proceso, Estructura y Resultado, una, solo el Proceso y tres, solo el Resultado.

El incentivo se causa cuando la prestadora cumple los objetivos de desempeño. Cuando se trata de $\mathrm{P}$ y $\mathrm{P}$, los objetivos son de productividad porque establecen la cantidad de consultas, vacunaciones y procedimientos a realizar. Estos objetivos no se negocian entre las Aseguradoras y Prestadoras porque están pre establecidos 
por el Ministerio de Protección Social usando como base la Resolución 412 de 2000.

En los pocos casos cuando las Aseguradoras utilizan P4P mas allá de $\mathrm{P}$ y $\mathrm{P}$, los objetivos apuntan a cierta mezcla de la calidad (realizar menos de $15 \%$ de solicitudes de exámenes de laboratorio en consultas de medicina general; menos de 8 días en la oportunidad de atención a los pacientes de medicina general; mas de $90 \%$ de satisfacción del usuario; menos de $0,35 \%$ de hospitalización; menos de $10 \%$ de remisión a especialistas; hasta $4 \%$ de consulta por urgencias entre la población asignada; menos de $5 \%$ de hospitalización por hipertensión y diabetes entre el total de usuarios crónicos.) y productividad ( $75 \%$ de personas cubiertas y $75 \%$ de adheridas al programa. La mayoría de los incentivos apuntan a los procesos de atención, y solo excepcionalmente, a los resultados en salud de la población (por ejemplo, la satisfacción del usuario).

La comprobación del desempeño de las Prestadoras se hace individualmente y nunca se utiliza el torneo, o la comparación de desempeño de varias Prestadoras.

En el nivel ambulatorio, $\mathrm{P} 4 \mathrm{P}$ se utiliza en combinación con el pago per cápita (Decreto 4747 de 2007).

Se utilizan dos esquemas de pago del incentivo: la bonificación variable combinada con la multa y el descuento por no cumplir las metas acordadas.

Si el precio acordado es 100 pesos por persona, bajo el esquema de bonificación variable se pagan 95 pesos por adelantado. Al finalizar el año, se analiza el desempeño de la Prestadora y se ajustan las cuentas. Si el desempeño estaba dentro del intervalo establecido, se paga 5 pesos lo que completa 100 pesos. Si el desempeño fue insatisfactorio, no se paga nada más o incluso se pide a la Prestadora devolver hasta 5 pesos. En el peor caso, la Prestadora ganará solo 90 pesos. Es un incentivo positivo relacionado con la multa. Otro esquema es el descuento que se aplica a las actividades de PyP que la Prestadora no realizó.

La experiencia Colombiana valida la TCT: para aplicar P4P, se necesita cierta cercanía y dependencia mutua entre la Aseguradora y Prestadora. Se encontró que solo dos Aseguradoras estaban administrativamente independientes de sus Prestadoras, mientras que seis Aseguradoras tenían relaciones especiales con sus Prestadoras porque tanto Aseguradoras como Prestadoras pertenecían a los mismos dueños. 
Una sola Aseguradora (CPS) manifestó utilizar P4P en el nivel hospitalario. El mecanismo consiste en el aumento o disminución del volumen de compra de servicios. Una vez al semestre CPS evalúa el desempeño de sus prestadoras hospitalarias utilizando los indicadores de satisfacción del usuario, ausencia de complicaciones, la disposición a servir. Los indicadores reflejan los procesos, y solo un indicador (satisfacción) refleja los resultados en salud. Luego CPS trata de direccionar a los usuarios hacia las prestadoras de mejor desempeño. La capacidad de direccionar a los usuarios no es grande porque CPS tiene pocas alternativas para reubicarlos. Esta capacidad es nula en el caso de las urgencias. Este tipo de relación es una clásica relación de mercado, bajo la cual el cliente varía el volumen de sus compras de acuerdo con su satisfacción con el proveedor. Como el cliente no paga más por la calidad, esta relación no puede ser clasificada como P4P.

\section{DISCUSION}

P4P en Colombia se usa exclusivamente por Aseguradoras del régimen Contributivo, solo en el nivel ambulatorio y básicamente en PyP. Es un resultado inesperado porque va en contra de la practica internacional (1-3).

Los resultados sugieren que el nivel ambulatorio es un territorio atractivo para experimentar con $\mathrm{P} 4 \mathrm{P}$ porque es la puerta de entrada al sistema, cuyo control es fundamental para controlar los costos totales. Especialmente atractivo es Prevención y Promoción porque en esta área no se necesita diseñar los objetivos ni indicadores de desempeño que están pre establecidos por Min. Protección Social.

En otras aéreas de servicio de salud esta ventaja no existe. El nivel ambulatorio tiene otro atractivo: la existencia de las alianzas estratégicas entre las Aseguradoras y Prestadoras. La experiencia Colombiana parcialmente valida la TCT. La TCT afirma que la forma Hibrida de gobernación presenta un nivel más alto de control administrativo y un nivel más bajo de incentivos que el Mercado como forma de gobernación. Efectivamente, se evidenciaron las relaciones de alianza entre las Aseguradoras y Prestadoras ambulatorias que permiten combinar un alto control con unos incentivos bajos o moderados. La evidencia a favor de la TCT es solo parcial porque las Aseguradoras no utilizan todas las oportunidades ofrecidas por la forma Hibrida de gobernación y practican P4P básicamente en P y P. Parece que la forma Hibrida no necesariamente abre el camino para $\mathrm{P} 4 \mathrm{P}$ y que la aplicación de $\mathrm{P} 4 \mathrm{P}$ depende también de otros factores, como la capacidad gerencial. 
En este mismo orden de ideas es oportuno preguntar ¿Por qué las Aseguradoras no utilizan P4P en el nivel hospitalario donde se originan $90 \%$ de sus facturas por los servicios? La TCT sugiere que las Aseguradoras, respondiendo a una mayor incertidumbre de la transacción hospitalaria, deberían utilizar la forma Hibrida de gobernación con los hospitales. La realidad es distinta: en la transacción hospitalaria domina el mercado. Parece que la incertidumbre de la transacción no determina la forma de gobernación. La creación de la forma Hibrida en el nivel hospitalario se encontró impedida por el bajo volumen de compra de servicios por cada Aseguradora. Como el sector de aseguramiento está fragmentado, una aseguradora importante como CPS no consume sino 10-15\% de la capacidad instalada de un hospital de alta complejidad. Parece que las consideraciones de economía de escala son fundamentales a la hora de determinar la forma de gobernación de la cual depende P4P.

Los resultados sugieren que P4P no se usa en el Régimen Subsidiado por varias razones. Primero, los recursos de contratación en salud del Régimen Subsidiado provienen en gran parte de los municipios que demoran con el pago. Segundo, en municipios pequeños existe el poder monopólico de las Prestadoras públicas municipales que imponen condiciones en la contratación. Tercero, el que realiza contratos del Régimen Subsidiado, es el alcalde, quien hace parte de la juta directiva de Prestadores municipales. Cuarto, las Aseguradoras Subsidiadas, que atienden población de bajos recursos y manejan el Plan Obligatorio de Salud Subsidiado (POSS), no tienen posibilidad de pagar más por el servicio de calidad.

Algunas Aseguradoras Subsidiadas utilizan el esquema de descuento que no castiga la baja calidad sino estimula la productividad. Cuando se aplica el descuento, la Aseguradora no paga por las actividades de P y P que la Prestadora no realizó. Se trata de una simple glosa de facturas o una curiosa mezcla de pago por cápita con el pago por evento que no constituye P4P. Los descuentos por las actividades no realizadas de $\mathrm{P}$ y $\mathrm{P}$ son cuestionables también desde la perspectiva misional. Quien debe inducir la demanda de servicios de prevención, es la Aseguradora. Cuando la Aseguradora contrata P y P bajo la modalidad de P4P, ella descarga su propia responsabilidad y la pone sobre los hombros de la Prestadora.

Los resultados hacen pensar que una de las causas del no uso de P4P en el nivel hospitalario radica en la incapacidad de las Aseguradoras de estandarizar la práctica médica. El intento de reducir la variabilidad de la práctica médica no va más allá de implantar guías médicas lo que no cambia la situación: cada usuario sigue siendo un caso especial, y cada hospital lo es con más razón. Es un obstáculo difícil de superar incluso para los entusiastas de P4P. 
Las limitaciones del presente trabajo derivan de una baja tasa de aceptación de participar en la investigación por parte de las Aseguradoras. Por ejemplo, Saludcoop, la Aseguradora colombiana más grande, se negó a participar

\section{REFERENCIAS}

1. Frolich A, Talavera J, Broadhead P, Dudley A. A behavioral model of clinician responses to incentives to improve quality. Health Policy. 2007; 80:179-193.

2. Petersen L, Woodard L, Urech T, Daw C, Sookanan S. Does Pay for Performance improve the quality of health care? Annals of Internal Medicine. 2006; 145: 265-272.

3. Van Herck P. Systematic review: Effect, design choices, and context of pay-for-p erformance in health care. Health Services Research. 2010; 10 (247):1-13.

4. Craig E. (Ed.) Routledge encyclopedia of philosophy. London: Routledge; 1998.

5. Smith A. Riqueza de las naciones (1776). Madrid: Alianza; 1994.

6. Taylor F. Principios de la administración científica. México: Herrero; 1976.

7. Prendergast C. The provision of incentives in firms. Journal of Economic Literature. 1999; 37: 7-63.

8. Ouchi W. A conceptual framework for the design of organizational control mechanisms. Management Science. 1979; 25: 833-848.

9. Boulton P, Dewatriport M. Theory of incentives, information, economic institutions. Cambridge: MIT Press; 2005. p. 318.

10. Institute of Medicine. Rewarding provider performance. Washington, DC. National Academies Press; 2007. p. 51.

11. Fehr E, Rockenbach B. Detrimental effects of sanctions on human altruism. In: Dewatripont $M$. The economics of contracting: foundations, applications and empirical investigations. Paris: DeBoeck; 2006. pp. 233-243.

12. Benabou R, Tirole J. Intrinsic and extrinsic motivation. In: Dewatripont M. The economics of contracting: foundations, applications and empirical investigations. Paris: DeBoeck; 2006.

13. Douglas C, Christianson J. Penetrating the "black box". Financial incentives for enhancing the quality of physician services. Medical Care Research Review. 2004; 61: 37S70 s.

14. Flamholtz E. Effective management control. Theory and practice. Boston: Kluwer; 1996.

15. Grossman S, Hart $O$. The costs and benefits of ownership: a theory of vertical and lateral integration. The Journal of Political Economy. 1986; 91 (6): 907-929.

16. Grant R. Ethics and incentives: a political approach. The American Political Science Review. 2006; 100 (1): 29-39.

17. Williamson $\mathrm{O}$. Comparative economic organization. The analysis of discrete structural alternatives. Administrative Science Quarterly. 1991;36:169-296. 